\title{
Towards Unification of Fundamental Interactions Using Non-Local Hidden-Variable Theory
}

\author{
Dirk J. Pons \\ Department of Mechanical Engineering, University of Canterbury, Christchurch, New Zealand \\ Email:dirk.pons@canterbury.ac.nz
}

How to cite this paper: Pons, D.J. (2020) Towards Unification of Fundamental Interactions Using Non-Local Hidden-Variable Theory. Journal of Modern Physics, 11, 1598-1619.

https://doi.org/10.4236/jmp.2020.1110100

Received: September 2, 2020

Accepted: October 19, 2020

Published: October 22, 2020

Copyright $\odot 2020$ by author(s) and Scientific Research Publishing Inc. This work is licensed under the Creative Commons Attribution International License (CC BY 4.0).

http://creativecommons.org/licenses/by/4.0/

\section{(c) (i) Open Access}

\begin{abstract}
Problem: In principle there might be a single deeper mechanism underlying the fundamental interactions at both the extremely small scale of particles, and the large scale of gravitation. However it is unclear what form such a theory might take, as the obvious candidates have not yet been successful. Purpose: This work constructs a conceptual framework for the interactions from a non-local hidden-variable (NLHV) perspective, specifically the Cordus NLHV theory. Findings: All the interactions can be attributed to the discrete force emissions from the particle, more specifically from the different attributes thereof. Thus the electrostatic appears to arise from the direct linear effect of the discrete forces; magnetic from bending of the flux tube; gravitation from handed energisation sequence; strong from the synchronisation of emissions; and weak from rearrangement of discrete force emissions hence remanufacturing of particle identity. Originality: An explanation is provided for all the interactions based on non-local hidden-variable theory. Apart from the concept of the discrete force, and its multiple attributes, no new particles or bosons are required.
\end{abstract}

\section{Keywords}

Field, Interaction, Fundamental, Quantum

\section{Introduction}

The concept of force is well established in mechanics and the resulting principles of statics and dynamics extensively applied in science and engineering with great success. However, despite the precision and familiarity with force at the macroscopic level, the fundamental physics of force are incompletely understood. 
Furthermore there is no single coherent theory for all the fundamental interactions. This paper proposes mechanisms for the deeper common causality between interactions. It approaches this from the perspective of a non-local hidden-variable (NLHV) theory [1] [2].

\section{Existing Approaches}

The first problem is explaining how force arises. The effects of electrostatic, magnetic, and gravitational (EMG) forces are well represented by Newtonian and classical continuum mechanics. However these mechanics do not describe the underlying mechanisms of how force arises or operates. The same limitation applies for general relativity (GR). In quantum mechanics (QM) force is conceptualised as occurring by the exchange of smaller particles, the gauge bosons. However there are many unexplained processes, such as how intrinsic properties are transferred between particles. Furthermore the bosons can only be detected as forces not individual particles.

The second problem is the lack of a single coherent theory for explaining all the fundamental force interactions. The interactions-excluding hypothetical dark energy mechanisms-are the electrostatic, magnetic, gravitational, weak, and strong. The standard model of QM proposes that electromagnetism is carried by virtual photons, the strong interaction between quarks by the gluon, and the weak interaction (e.g. quark flavour-changing between left-handed fermions) by $\mathrm{W}$ and $\mathrm{Z}$ bosons. The strong nuclear force has a partial explanation in quantum chromodynamics (QCD), but the theory is limited to quarks. It also has no explanation of nuclear structures at the level of nucleons and atomic nuclei. A coherent explanation of gravitation is especially problematic from a particle perspective. In GR, gravity arises from the warping of space time, i.e. the effect is a geometric one [3]. Holographic theory provides an explanation for gravitation as an entropic force [4], but not for the deeper mechanisms or the other forces. It seems that any theory that explains gravitation does not explain all the other interactions. QM has attempted to explain gravitation by loop quantum gravity, but a solution is elusive. Alternatively, gravitons may be the boson for gravity, though this is speculative. Thus there is no accepted explanation within QM for gravitation.

Existing efforts at unification have primarily attempted to extend quantum mechanics, on the expectation that a continuum physics like general relativity is unsuited to representing particle interactions. A second premise that has historically shaped the research is the belief that there ought to exist an undiscovered single progenitor force that, at sufficiently high energy density, forms the basis for all the other interactions. Unification is indeed available for the electrostatic and magnetic forces into the electromagnetic with the photon as the boson, and decay interactions (weak nuclear force) via electroweak unification. Models exist providing grand unified theories whereby the electromagnetic, weak, and strong forces might unite into an electronuclear force. However the predicted new particles have not been observed. Furthermore QCD is not integrated with electroweak theory, and gravitation has been especially problematic. 
The elusiveness of solutions may indicate that the necessary physics is not accessible with the premises that underpin quantum mechanics. QM is premised on particles-and bosons being zero-dimensional (0-D) points with stochastic and intrinsic properties. If the interactions were to actually have a spatial component, then $0-\mathrm{D}$ points might be inherently unable to represent the mechanisms. Hence alternative theories of physics are also candidates, with their greater number of dimensions for their particles. These other theories may be broadly categorised into string theories, and hidden variable theories. Neither has been especially effective.

String theory has been attempted as a route to a theory of all the interactions. However its lack of specificity has frustrated progress. Hidden variable theories take the premise of physical realism, that manifest attributes are carried by physical substructures to the particle, but they too have been mostly unsuccessful. Of the NLHV theories, historically the de Broglie-Bohm has been the best known [5] [6], but has made no contribution to a holistic explanation of force interactions. Hence a unified theory of all interactions remains elusive, despite considerable effort.

\section{Method}

The purpose of this paper was to seek explanations for each of the interactions under the Cordus NLHV theory [1]. The earlier work of [7] and [8] provided the initial conceptual basis.

The approach used abductive reasoning, which starts with the known observations and seeks to find the simplest mechanisms that will account for the phenomena consistent the existing premises. "Simple" in this context was interpreted as parsimonious and sufficient, while "Consistent" refers to system integration.

- Parsimoniousness-New particle sub-structures should not be created for each new phenomenon (function) being included. This is conceptually similar to the need to avoid over-parameterisation and model degeneracy in quantitative modelling.

- Sufficiency-The solution needs to be a sufficient match to the empirical phenomena. This corresponds to the function needs in the language of design, see also [9].

- System integration-Any newly proposed particle substructures or concepts must be integrated into the rest of the theory. This is necessary for conceptual coherence. Where appropriate this is expected to result in reconsideration of parts of the theory that show conceptual discoherence.

This approach was applied iteratively. The results present the final theory that emerged, not the intermediate work. The theory is constructed of lemmas, indicated $\S$, which are conceptual propositions that are valid within the wider Cordus theory, even if unable to be immediately verified. The abductive reasoning process is represented diagrammatically in Figure 1. 


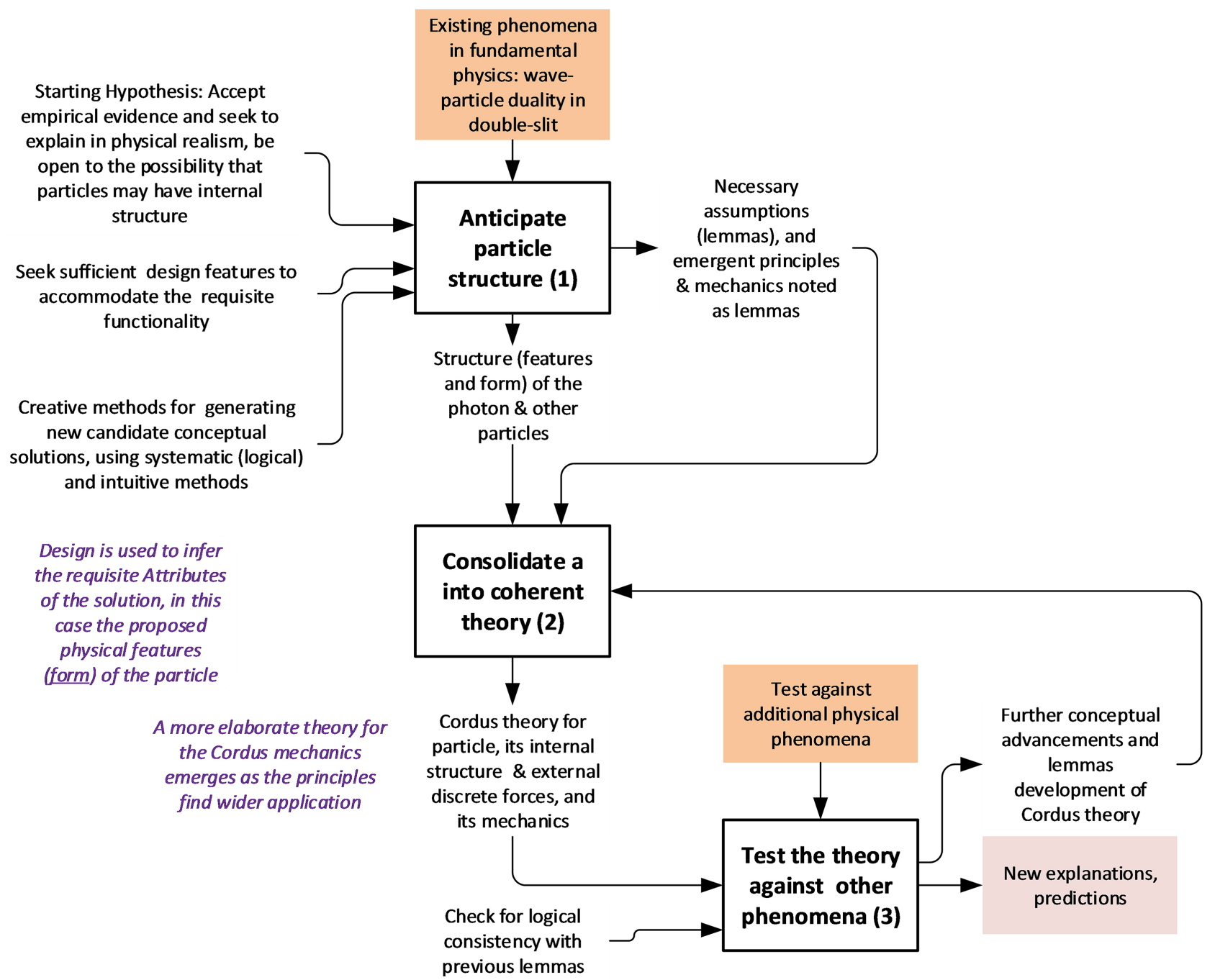

Figure 1. Flowchart representing the abductive reasoning method as applied to this theory.

We first present general principles for interactions, and then elaborate on the different sub-types of interaction.

\section{Results}

\subsection{Context}

\subsubsection{Structure of the Cordus Particle}

Like any NLHV theory, the Cordus theory proposes that particles are not zero dimensional points but instead have internal structures that provide the observed functionality (charge, mass, spin, etc.). It makes specific predictions about the identity of these structures at the sub-particle level [1]. The key difference compared to other theories is that the particle is proposed to comprise two reactive ends some short distance apart, connected by a fibril, see Figure 2. The fibril does not interact with other particles, instead the interaction occurs at the reactive ends. The reactive ends are energised at the de Broglie frequency, at which time they emit discrete forces into space in each of three spatial directions (r, a, 
and t, see Figure 2) [10] [11]. For massy particles the discrete forces are encapsulated into extended fibril structures that propagate into space-these are called flux tubes. The aggregation of these in space makes up a fabric of discrete forces [12].

\subsubsection{A Proposed General Mechanism for Force}

A general mechanism for force has been proposed within this theory [13]. The reactive end moves as it goes through its energisation cycle, and traces out a cyclic locus in space. This locus is then distorted by the incoming discrete forces of the external field. As a consequence the mean position of the reactive end changes. This provides a mechanism whereby the reactive end is able to sample the spatial distribution of field around it, i.e. the gradient of the field, and move accordingly. The direction of motion adjustment is along the gradient in the direction of greater compatibility of discrete force emissions. Generally this means

\section{Electron e}

Characterised by one discrete force in each of the three directions. This balanced loading causes the structure to be stable against decay.

Physical structure

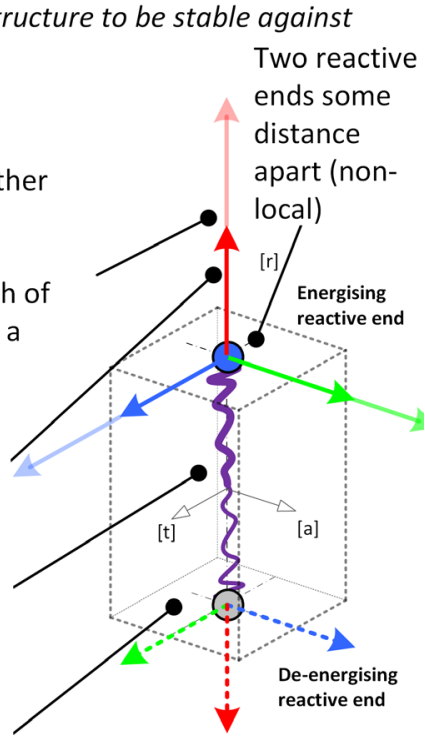

The discrete forces are released rather than retained as in the photon. Consequently there is an enduring succession of discrete forces in each of the three directions, which creates a long-ranged force effect.

New discrete forces continue to be created and sent down the flux tube at each frequency cycle Inner Fibril provides instantaneous communication between reactive ends, hence a non-local effect

Type of reactive end: pulsatile. One reactive end energising and the other de-energising $\left(180^{\circ}\right.$ out of phase)

Notation
The HED notation represents the
distribution of the discrete forces
in the three emission directions
(HEDs)
Three orthogonal axes
$(r, a, t)$ for emission of
discrete forces

Dexter hand of energisation sequence for matter: red $\rightarrow$ green $\rightarrow$ blue. For the energising end this is $[\mathrm{r}] \rightarrow[\mathrm{a}] \rightarrow[\mathrm{t}]$.

Each discrete force carries

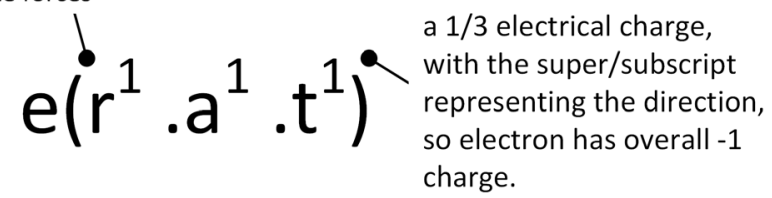

Figure 2. The representation of the electron's internal and external structures. It is proposed that the particle has three orthogonal discrete forces, energised in turn at each reactive end. Reproduced from [13], being an adaption of [14] with permission. 
the type of emissions, e.g. the phase energisation sequence (matter-antimatter), and direction of emission (charge). Hence force arises as "coerced displacement of the reactive end" [13].

\subsubsection{Geometric Properties of Flux Tubes Correspond to Interactions}

The flux tube has three geometric properties of linear strength (radial emissions), curvature (bending), and twist (torsion). It is proposed that these geometric properties provide the mechanisms for the electrostatic, magnetic, and gravitational (EMG) force interactions respectively. This concept arises from [7] and [8], and is further elaborated below. A fourth attribute of the discrete forces (not so much of the flux tube) is the synchronisation of emission between two particles [10], and this is proposed as the mechanism for the strong interaction (see below). The weak (decay) interaction is proposed to have an altogether different cause (see below).

The above are the general principles for how force operates within the Cordus theory. Next these principles are applied to offer explanations for the different interactions.

\subsection{Electrostatic Interaction}

Consider test particle B, say an electron, in an electrostatic field set up by basal particle $A$. Each particle has two reactive ends, and for $B$ these are denoted $B_{1}$ and $B_{2}$. See Figure 3. The location of interest is reactive end $B_{1}$ which receives forces from $\mathrm{A}$, and emits its own discrete forces.

\subsubsection{The Electrostatic Interaction arises from the Linear (Radial) Component of Discrete Forces $(\$ 1)$}

The theory proposes that the electrostatic interaction arises from the direct linear action of the incoming discrete force on a remote particle. It applies in a direction along the line of the flux tube, and the effect is to move the remote particle B closer or further from the emitting particle A depending on the relative charge.

The underling mechanism is proposed to be that the recipient reactive end attempts to emit its own discrete forces, but is affected by the incoming discrete force. It may be enhanced or inhibited depending on the compatibility between the native and external discrete forces. This changes the position of the reactive end, with the displacement being along the axis of the fibril. A similar mechanism for the spatial readjustment of the reactive end has been proposed for photon absorption and emission [15]. The direction of the discrete force is the basis for the sign of the electric charge. This is presumed to be the direction of action of the discrete forces within an outward travelling flux tube. Negative charge is assumed outwards - this is merely a sign convention.

\subsubsection{Explanation for Like-Charge Interaction}

Consider the interaction between two electrons, being a typical like-charge case. Electron $A$ is the basal one and is nominally assumed to be fixed, while $B$ is the remote test charge that reacts to A. Each electron has two reactive ends (1 and 2) 


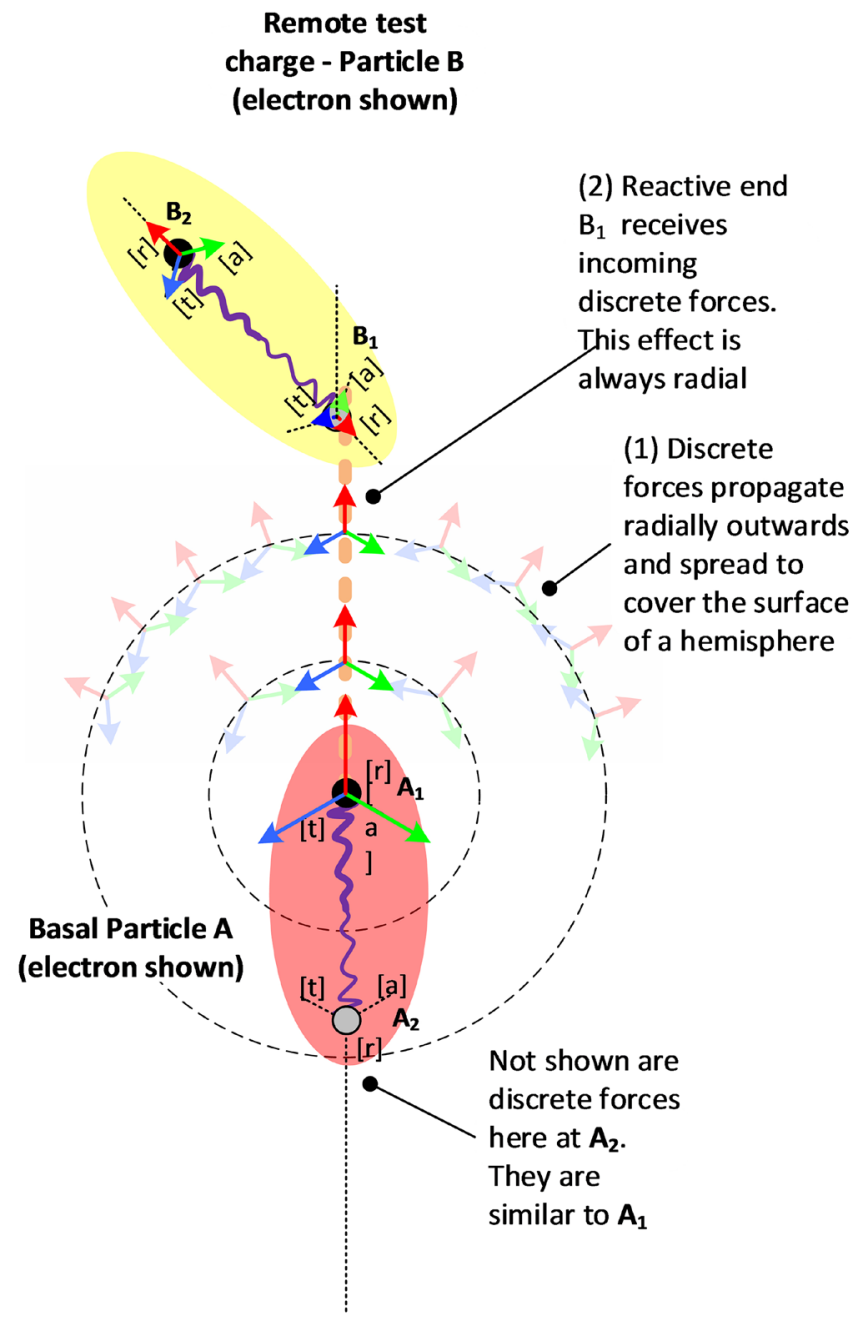

Figure 3. The electrostatic interaction arises from the direct linear action of discrete forces emitted by basal particle A.

per this theory. The reactive end $A_{1}$ of electron A emits discrete forces packages in a flux tube, which propagate out into space at the local speed of light and impinge on reactive end $B_{1}$ of remote test electron $B$.

Consider now the energisation of dormant reactive end $B_{1}$. As $B_{1}$ begins to energise it encounters incoming discrete forces from $A_{1}$ that are incompatible with its own emissions. Per lemma 1, this ranged interaction is transphasic, since the particles have like charge, and the synchronous interaction therefore operates to put the emissions $\pi / 2$ out of phase. However $B_{1}$ cannot delay its energisation indefinitely. This is because energisation state and frequency are determinants of particle identity and energy respectively [16] [17]. Consequently $B_{1}$ has to adjust its location to move with those imposed forces, in the repulsive direction.

If the remote charged body has physical depth, i.e. comprises many particles, then the incoming discrete forces apply displacements to the foremost parts of the body, then pass through and apply displacements to the deeper layers. Hence a charged macroscopic object feels the electrostatic effect and moves holistically. 


\subsubsection{Electric Field}

At the macroscopic scale of a negatively charged sphere, each of many electrons generates its own discrete forces and flux tubes. Macroscopic bodies tend not to exhibit quantum behaviours. The Cordus theory interprets such bodies as discoherent assemblies of matter, wherein the particle spans are not aligned [2]. Hence a discoherent sea of electrons can be expected to generate flux tubes pointing in random directions. This is consistent with the observation of an electrostatic field that is smooth, continuous, and uniform in all directions. The theory predicts that the field will however be granular at the frequency of the charge emitters.

The electrical force $\boldsymbol{F}_{e B}$ between two particles of charge $q_{A}$ and $q_{B}$ is:

$$
\boldsymbol{F}_{e B}=-K \frac{q_{A} q_{B}}{r^{2}} \hat{r}
$$

where $r$ is the radial separation, $\hat{r}$ is the radial direction and $K$ is a constant. A qualitative explanation for the form of this relationship follows. The reason for the product of charge $\left(q_{A} q_{B}\right)$ is that charge determines the number of discrete forces involved: the amount of displacement coercion (hence force) experienced by particle B is determined by the strength $q_{A}$ of the incoming discrete forces, and the strength of its own response $q_{B}$, hence a multiplicative relationship. The inverse square relationship $1 / r^{2}$ arises from the expansion of the discrete forces on a spherical front. This expansion occurs because the discrete forces are emitted in three orthogonal directions in surface shells at each energisation cycle.

Irrespective of the relative orientation (spin) of $\mathrm{A}$ and $\mathrm{B}$, there is always a component of B's emissions that is radial to A, i.e. in the direction $\hat{r}$. The electrostatic interaction may be simplified to only this component.

Dissimilar charges attract even across the matter-antimatter species. This implies that the energisation sequence-which differentiates the species-is immaterial for the electrostatic interaction.

\subsubsection{Electrostatic Shielding}

An electric field is known to be shieldable by a Faraday cage, whereas gravitation is not. The present theory predicts that discrete forces penetrate all matter, but in a Faraday cage the electric field only appears to be shielded, because electrons in the conductive cage material have sufficient mobility to move in response to the external field and set up a countering field.

In contrast the photon can be shielded: it can be absorbed, by mechanisms identified in [15]. Applying this to interaction of electromagnetic radiation photons from an antenna or reflector, the present theory explains that the frequency and span of the photons is inversely related [1], which is consistent with the observation that the conducting elements of the antenna need to be closer spaced for higher frequency radiation. As the photon frequency rises still further, the required conductive loops are of the order of atomic spacing, i.e. the shield must be of a continuous material. For even greater frequencies the electrons cannot 
encounter all the discrete forces in which case the photons pass straight through.

\subsection{Magnetic Interaction}

A test charge $q$ moving with velocity $V$ in a magnetic field $B m$ experiences a sideways force $F_{m}$ that is perpendicular to both its direction of travel and the $e x$ ternal magnetic field, i.e. excludes the magnetic field of the test charge itself):

$$
F_{m}=q V \times B m
$$

This is explained as follows.

\subsubsection{Movement of the Basal Charge Bends the Flux Tube ( $\$ 2)$}

A flux tube is a directional propagation of discrete forces. It is proposed that this may be bent by movement of the basal emitting reactive end. Per Lemma 8 , motion of a reactive end occurs in discrete displacements during its de-energised state. This results in emissions expanding from each progressive location of energisation. Consequently the flux tube, which is continuous, has kinks. The new curvature moves outward with the discrete forces at the local speed of light. If the speed of light was infinite, i.e. disturbances propagated instantly, then there would be no magnetic effect.

\subsubsection{The Magnetic Interaction Arises from Curvature of the Flux Tube}

Under these premises the magnetic interaction arises interpreted as the action of the incoming discrete forces in a recurved flux tube, causing a yaw adjustment in the velocity of a remote moving charged particle [7]. A remote test particle B moving with velocity $V_{B}$ receives at reactive end $B_{1}$ the discrete forces and the re-curved flux tube emitted by $A$. This causes a coerced displacement of $B_{1}$, which provides a yaw moment that changes the direction of motion of $B$ to be more parallel or antiparallel (depending on the charge) to the velocity of A. See Figure 4.

\subsubsection{Coerced Displacement of a Remote Moving Charge}

How does a curved flux tube create the magnetic force on the moving remote test charge? If the remote test charge is stationary, then any curvature of the incoming discrete forces in their flux tube only re-orients the direction of the electrostatic force, i.e. momentarily changes spin. Since the flux tube is recurved, this imposes a transient change in orientation that integrates to zero. However, if the test charge is also moving, and encounters a curved flux tube, then the magnetic interaction attempts to realign the locus of the moving test charge to the same handed direction of motion as the basal charge.

When the discrete force in its flux tube reaches the remote moving test charge $B$, it upsets the geometric location for the reactive end $B_{1}$ of the moving test charge. Whether it delays or advances that reactive end depends on the sign of the magnetic field, i.e. the relative direction of the velocity of $\mathrm{B}$, and the relative charges. The discrete force pulse prevents $B_{1}$ from advancing forward as far as it usually might during a frequency cycle, or it pushes it forward. 


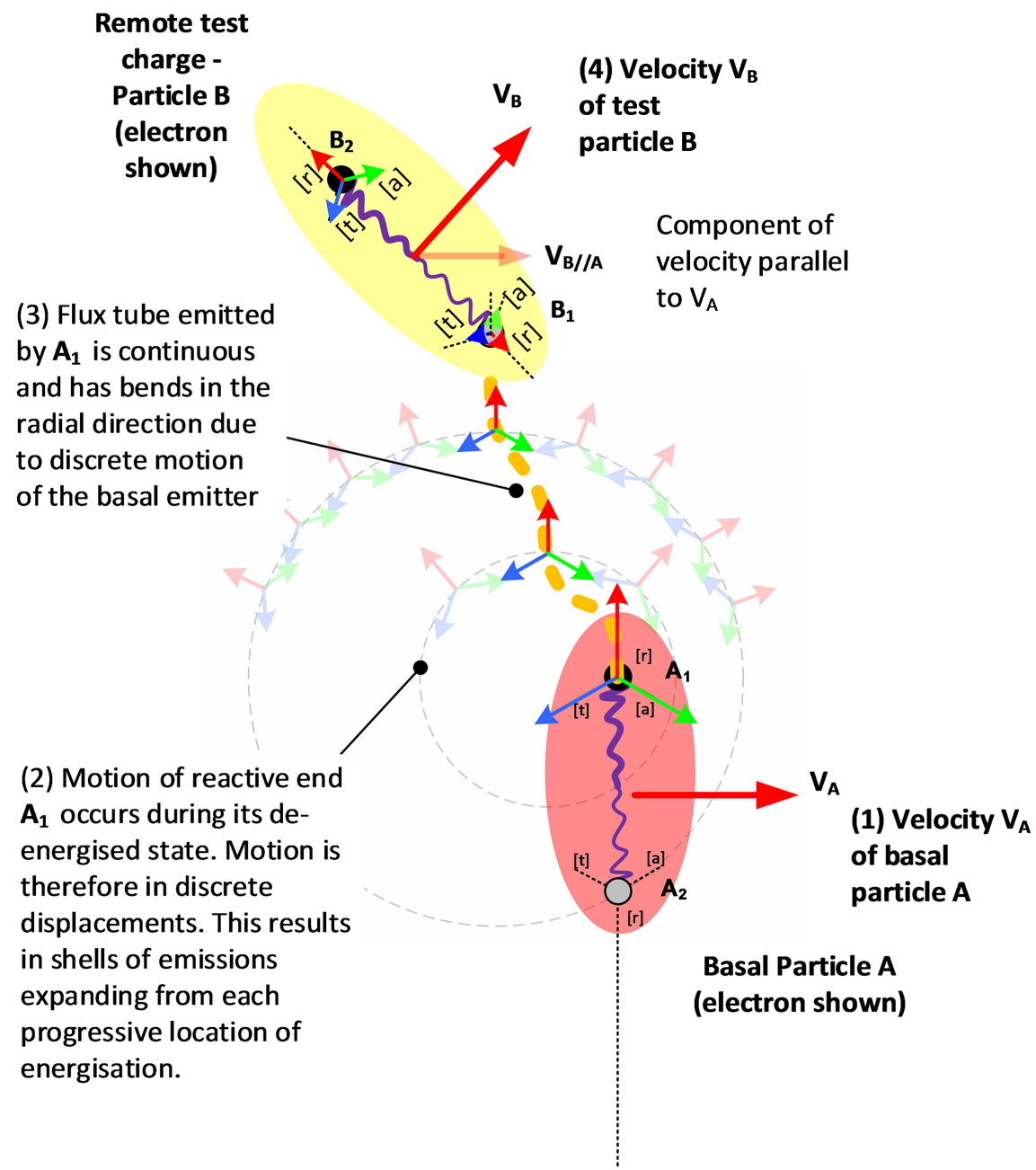

Figure 4. The magnetic interaction arises from the rotational action of the incoming discrete forces in a recurved flux tube.

The incoming discrete forces are not consumed but pass on outward to the other reactive end of the remote particle. The effect on $\mathrm{B}$ as a whole is additive rather than being negated, since the direction of the flux tube is reversed when it reaches its other reactive end. This sets up a yaw moment across the fibril of the remote particle, thereby adjusting the direction in which the remote charge is moving.

\subsubsection{Magnetic Fields}

Magnetic fields represent the motion of the charge (basal generator) that is emitting the discrete forces. A remote moving charge changes direction to be more closely parallel (or antiparallel depending on charge sign) to the locus of the basal charge [18]. The magnetic force $\boldsymbol{F}_{m B}$ in the radial direction $\hat{r}$, experienced by particle B of charge $q_{B}$ and velocity $v_{B}$ in a magnetic field created by particle A with $q_{A}$ and $\boldsymbol{v}_{A}$ is:

$$
\boldsymbol{F}_{m B}=\frac{\mu_{0}}{4 \pi} \frac{q_{A} q_{B}}{r^{2}} \boldsymbol{v}_{A} \times \boldsymbol{v}_{B}
$$


where $r$ is the radial separation, and $\mu_{0}$ is a constant. A qualitative explanation for the form of this relationship follows. The reasons for the product of charge $\left(q_{A} q_{B}\right)$ and the inverse square relationship $1 / r^{2}$ are as before. Having more charges $q$ moving in the same direction does not increase the curvature but simply means that there are more discrete forces reaching the remote test charge, i.e. the effect simply scales for increase in either of the charges. The direction of the magnetic field is perpendicular to the plane in which the curvature of flux tube occurs. The velocity dependency arises because the faster the basal charge moves $v_{A}$ the greater the curvature of the flux tube. The greater the velocity $v_{B}$ the greater the rate at which the kinks are encountered, hence a multiplicative relationship. The magnetism effect depends not simply on the speed of the charges, but also their relative directions. The effect depends on the component of curvature that is apparent to $\mathrm{B}$ in its direction of motion, hence the cross product. Thus magnetism only works in remote particles that already have some degree of alignment of their locus with the velocity of the basal charge.

It is proposed that the reason for the effect being right-handed is due to the way that reactive ends emit discrete forces in an energisation sequence (see Figure 2), and the dominance of the matter species. Each package of discrete forces has three orthogonal sub-forces and these are energised in a sequence. This sequence has been proposed as the distinguishing structural feature of the matter-antimatter species [19], the anti-electron structure [20], and the basis for annihilation [21].

A common illustration of magnetism depicts a moving charge being forced into a circular trajectory in the presence of a uniform magnetic field. Our explanation is that when the magnetic field is large and uniform, then the transecting moving test charge is forced into a circular path which is the same motion as the large basal current required to make that magnetic field. In this case the magnetic field dominates the interaction, and the moving test charge tends to move into a circular or helical trajectory (its own back-reaction is miniscule). However the case of the uniform field obscures the important fact that creation of that uniform field requires charges to be moving in a circular path too. Uniform magnetic fields are therefore a special case. It is the interaction of two moving charged particles where the more interesting mechanics occurs. The geometrically simplest form of magnetism, two particles affecting each other, is surprisingly complex. The moving test charge is not simply a passive participant, but also radiates its own discrete forces in their flux tube. If the basal charge is of similar size, it will be affected in turn by the magnetism of the test charge. For this and relativistic considerations see [22] and earlier work by [18]. We propose it can be qualitatively interpreted as one moving charge attempting to force another to conform to the same direction of motion: it is a type of alignment effect.

If the test charge is not moving, then the effect of incoming magnetism is to align the remote test charge, which means interfering with its orientation (spin). 
This is consistent with behaviour of permanent magnets and magnetic resonance imaging. In both cases the spins of all the electrons in the macroscopic body tend towards alignment. Again this shows that magnetism is primarily an alignment effect.

\subsubsection{Permanent Magnets}

A permanent ferromagnet has a magnetic field, but no apparent electric field. The usual explanation is that that the electron and nucleon spins are aligned across a domain (region of atoms). The present theory explains this based on the orientation of the particle. It is proposed that the alignment of the span of electrons and nucleons, i.e. spin, results in the discrete forces in their flux tubes pointing in the same direction along the axis of the magnetic poles, but randomly orientated in the transverse directions. Hence the effect is summated along the axis, and neutralised laterally. The magnet does not appear to be charged or to emit an electric field because of the equal contribution of positive and negative charges. Nonetheless it emits discrete forces in their flux tube. The magnetic domains are proposed to be formed in the first instance because electron discrete forces extend to neighbouring atoms and encourage alignment of other electrons. We suggest that within the magnetic material the electrons themselves move, either through their unfilled orbitals, or as current flow within the sub-lattices of the material, and this generates curvature of the discrete forces in their flux tubes and thus magnetic fields.

\subsection{Gravitation}

\subsubsection{The Gravitational Interaction Arises from Torsion of the Flux Tube, Which Arises from the Handedness of the Emission of Discrete Forces ( $\$ 4$ )}

Up to here the discrete forces have been implied to be discrete, and represented diagrammatically as arrows. However this is a simplification of a deeper mechanics. They are instead believed to take the form $\sin ^{2}(\theta / 2)$ and to comprise emissions in three orthogonal directions ( $r, a, t)$ [13], see Equation (4). Thus the potential energy $U_{B_{1}}$ in the flux tube has three components offset at thirds of the phase angle $\theta_{B}$ :

$$
\begin{gathered}
U_{B_{1}}(r)=\sin ^{2} \frac{\theta_{B}}{2} \\
U_{B_{1}}(a)=\sin ^{2}\left(\frac{\theta_{B}}{2}-120^{\circ}\right) \\
U_{B_{1}}(t)=\sin ^{2}\left(\frac{\theta_{B}}{2}-240^{\circ}\right)
\end{gathered}
$$

See Figure 5 for a representation of these three phases for a matter species (electron). This energisation sequence results in a torsional effect on remote particle $\mathrm{B}$, which moves closer or further along the field gradient depending on the mutual compatibility. 


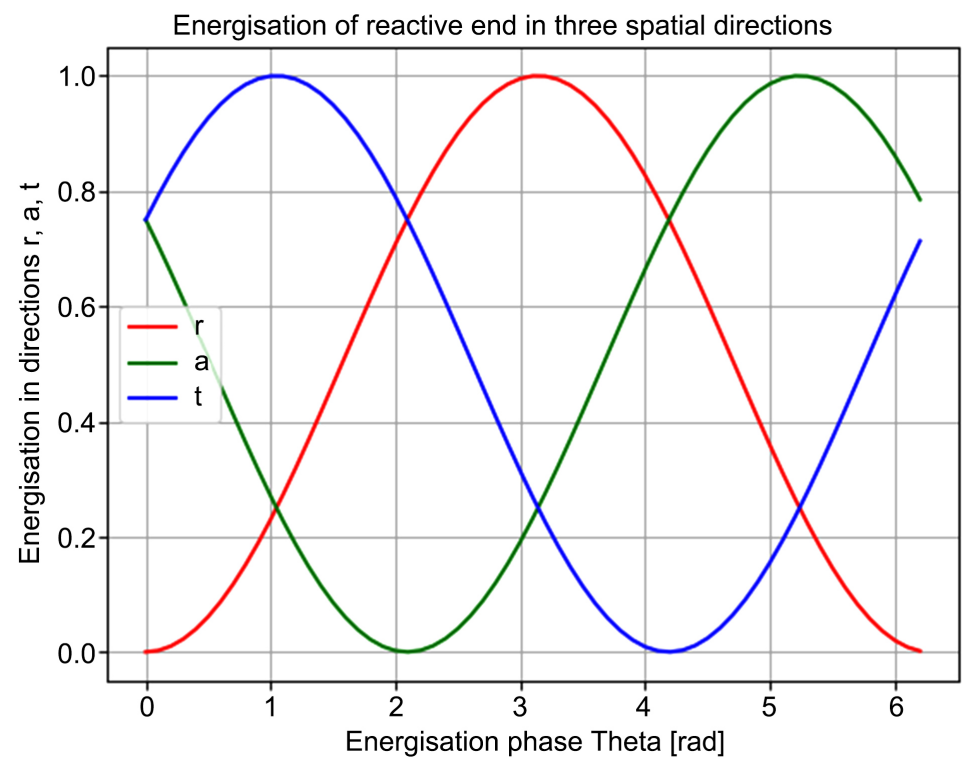

Figure 5. Energisation phases in the three orthogonal emission directions $(r, a, t)$ follow a $\sin ^{2}(\theta / 2)$ relation. This is for a matter particle (electron). Image from [13] with permission.

\subsubsection{Further Implications}

There are several implications. The first is that the reactive end is never fully de-energised. While any one discrete force does momentarily go to zero in strength, the reactive end as a whole maintains its energisation. This satisfies another part of the theory which showed that the Lorentz and relativistic Doppler could be derived on the premise of a stretchable flux tube [23].

A second implication is that the potential energy function for reactive end $B_{1}$ has a circular function, and the reactive end moves in reaction to trace a small cyclic locus in space [13]. The locus is circular with normal $[1,1,1]$ in the $[r, a$, t] directions. However when an external field is imposed, then the reactive end expands its excursion asymmetrically, and this moves its mean location.

A third implication concerns the question of why the gravitational interaction between matter and matter is attractive when the electrostatic interaction repels like charges. The answer is that for the electrostatic the opportunity for greater coordination between particles arises if their directions of emissions (charge) are opposite, irrespective of the energisation sequence (species). In contrast for gravitation the opportunity arises if the emissions have the same hand: receiving particle $B$ is attracted to the transphasic interaction [13] whereby it energises within the null points in the incoming $(r, a, t)$ emission. This it can only do if the energisation sequence is the same, which always arises for when both particles are matter species.

\subsubsection{Matter-Antimatter Gravitation}

In this theory the energisation sequence $(r, a, t)$ vs. $(a, r, t)$ determines the matter-antimatter species [19] [24]. Thus we predict that antimatter-antimatter gravitation is attractive, and matter-antimatter repulsive. The reasoning follows. For the case of an anti-electron the energisation sequence is $(a, r, t)$ and the charge is 
inverted. The corresponding energy locus for the reactive end is shown in Figure 6 for the electron and antielectron. This shows that the rotation is opposite in direction for antimatter. It logically follows that the gravitational interaction between two antimatter particles would be attractive, and repulsive between matter and antimatter.

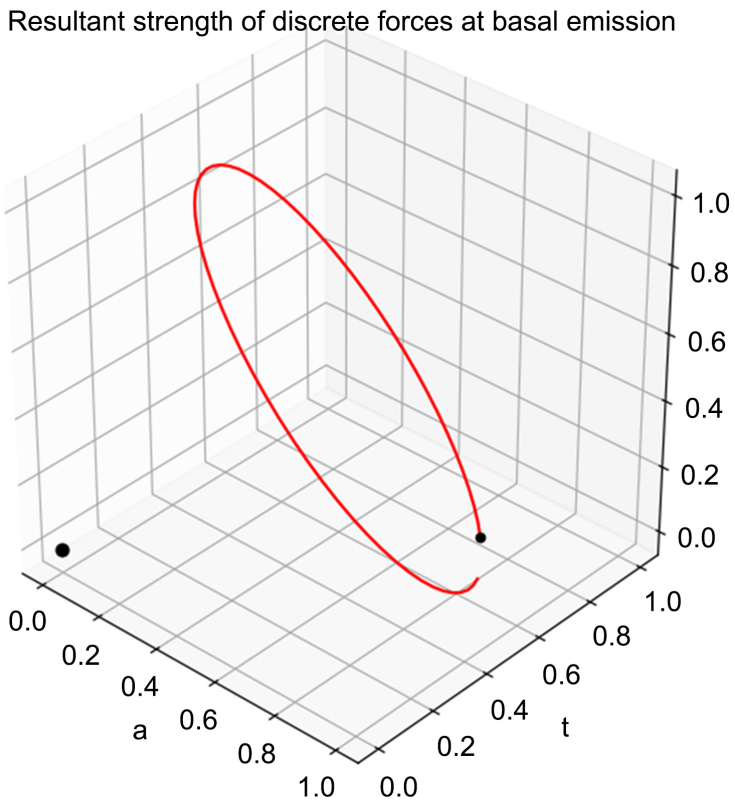

(a)

Resultant strength of discrete forces at basal emission

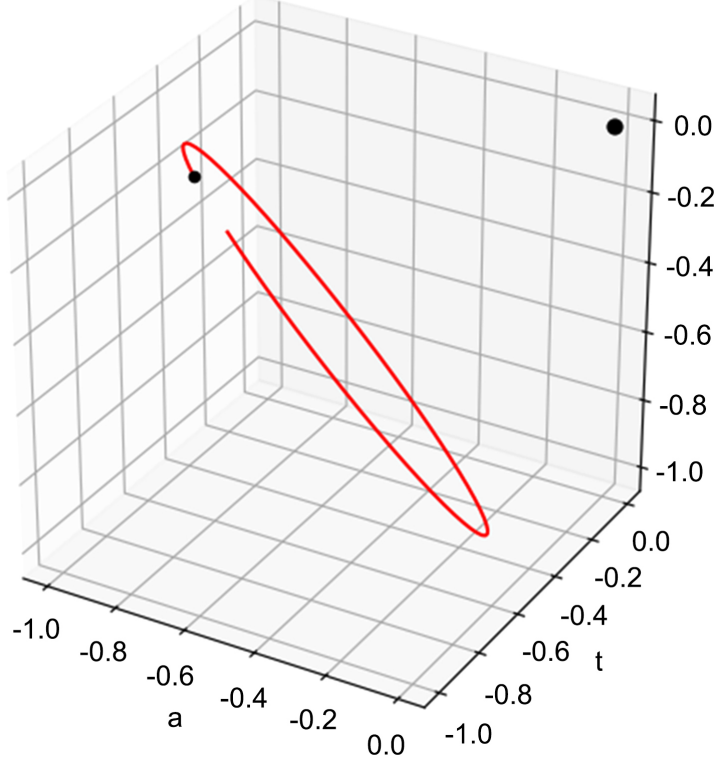

(b)

Figure 6. Resultant energy at the basal generator $B_{1}$ due to (a) matter negative charge (electron) from [13], and (b) antimatter positive charge (antielectron). The axes are ( $r$, a, $\mathrm{t}$ ). The larger black marker indicates the nominal origin $(0,0,0)$, and the smaller black marker indicates the location for $\theta=0$. The locus is deliberately shown incomplete to indicate the direction of rotation. 


\subsubsection{Gravitational Force}

The gravitational force $\boldsymbol{F}_{g B}$ in the radial direction $\hat{r}$, experienced by particle B of mass $m_{B}$ in a gravitational field created by particle A with $m_{A}$ is conventionally:

$$
\boldsymbol{F}_{g B}=G \frac{m_{A} m_{b}}{r^{2}}
$$

The reason for the product of charge $\left(m_{A} m_{b}\right)$ is that that the effect is enhanced by more incoming discrete forces $\left(m_{A}\right)$ per lemma 1 , and a greater frequency of response $\left(m_{b}\right)$ per lemma 8 . The inverse square relationship $1 / r^{2}$ is because of the dilution of discrete forces across an expanding spherical shell, as before.

\subsection{Synchronous Interaction (Strong Nuclear Force)}

The equivalent of the strong nuclear force in the Cordus theory is the synchronous interaction [10]. This interaction is between reactive ends from different particles, that are co-located. If their discrete force emissions are compatible, then this locks the reactive ends together. As identified in Lemma 2 [13], the synchronous interaction has two subtypes, which are transphasic and cisphasic. The terms refer to the phase difference at synchronisation.

The strongest form of compatibility is where the assembly provides a balanced and complete set of discrete force emissions in all three directions [25]. In this case the reactive ends energise simultaneously, and this is the cis-phasic subtype of the synchronous force. An example of the cis-phasic interaction is proposed in the bond between the proton and neutron in the atomic nucleus, see Figure 7.

The other subtype of synchronous interaction is trans-phasic, where the reactive ends from two separate particles energise out of phase. The type example of this is the Pauli electron pair [2], see Figure 8.

Application of the synchronous principle is able to explain the structure of the atomic nucleus as a polymer of neutrons and protons. Rules for these bonds have been deduced, and these are sufficient to economically explain why any nuclide is stable, unstable, or non-existent. This has been demonstrated for $\mathrm{H}$ to $\mathrm{Ne}$ [25] [26] and the trends appear to hold up to at least Ar.

Thus the nuclear force may be explained as a synchronisation of emissions between different reactive ends. The theory proposes that the strong force at the deeper quark level is likewise a synchronous interaction, though the internal structure of the proton and neutron have yet to be fully elucidated within this theory.

\subsection{Remanufacturing (Weak) Interaction}

Per QM the weak interaction mediates the nucleon beta decay and electron capture processes via exchange of $\mathrm{W}$ and $\mathrm{Z}$ bosons. From the Cordus theory the interpretation is somewhat different, as the analysis [16] suggests the weak interactions are part of a more general family of processes that change particle identity. From this perspective the weak bosons are merely transitional assemblies, rather 


\section{Approach of two particles (proton and neutron)}

Neutron and proton have different arrangements of their discrete forces, both the arrangement across the three axes, and the direction (charge)

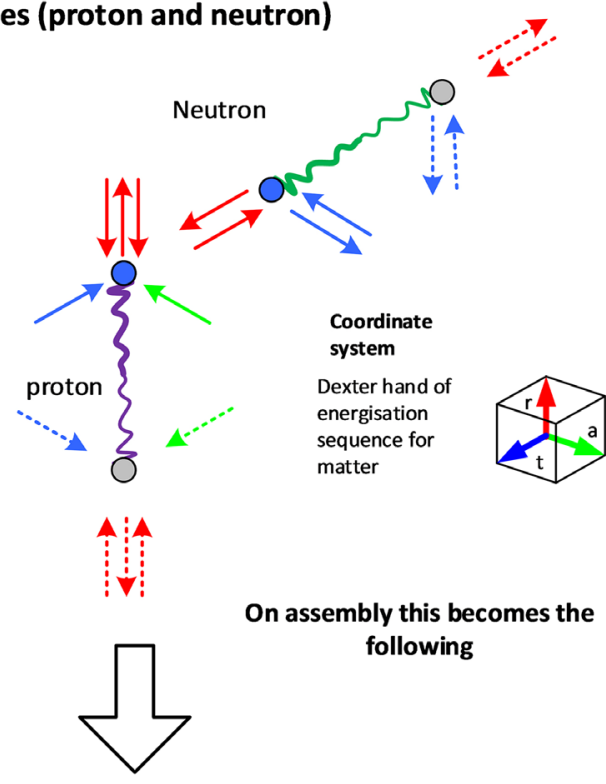

At assembly the two reactive ends co-locate, and synchronise the emissions of their discrete forces. This synchronisation holds the particles together, i.e. provides a bond or force.

The result is that the discrete forces are equal in each of the three axes, regardless from which particule they originated. This balance is proposed as the reason for stability.

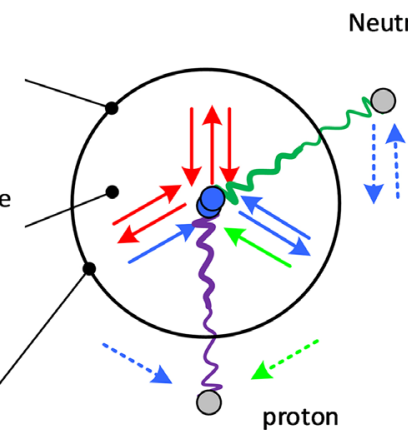

Neutron

The sum of the discrete forces in HED notation at the joint is $\left(\mathrm{r}^{1}{ }_{1.1} \mathrm{a}^{1}{ }_{1.1} \mathrm{t}^{1}{ }_{1.1}\right)$ which is : a full unit of positive charge

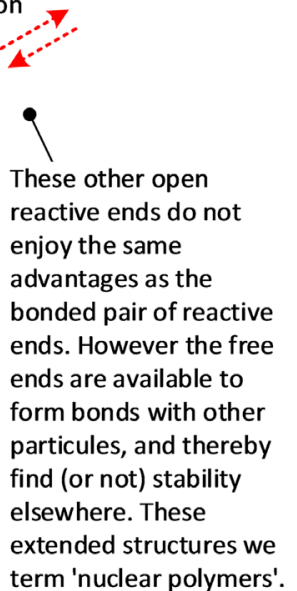

Figure 7. Key features of cis-phasic synchronous interaction as illustrated in the proton-neutron bond. Adapted from [10].

\section{Phase angle of re- energisation, $\theta=\pi$ for particules in a trans- phasic coherent assembly.}

The reactive ends of the two electrons look after each other's location in a complementary way

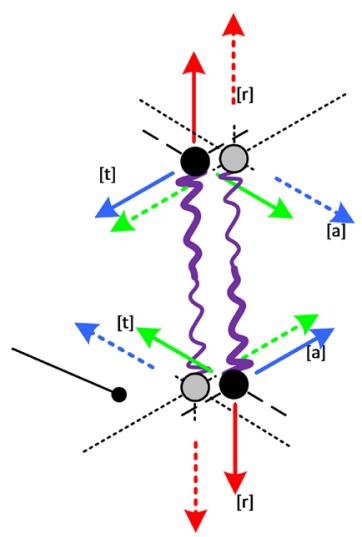

Figure 8. The Pauli pair of electrons uses the trans-phasic interaction to provide a degree of bonding. Adapted from [2]. 
than particles that cause change in quarks. Hence they do not deserve to be called bosons or their process singled out as a fundamental interaction. Instead we propose a set of principles of discrete force manipulation for the remanufacturing processes generally.

Particle identity, in the Cordus theory, arises from the characteristic pattern of discrete force emissions. Change these, and the particle identity is also changed. This process is called "remanufacturing" because the discrete forces are conserved. The conventional term "decay" implies a degenerative, disorderly, or destructive process, which from the Cordus theory is quite the wrong way to think about these transformations. Consideration of the discrete forces and the rules for their transformation [16] shows that it is necessary for many processes to change the order of energisation sequence. The theory shows that the neutrino species achieve this by removing unwanted handedness from the assembly [17]. Note that in this theory the handedness is also proposed as the matter-antimatter species differentiator [17] [21]. This is a further reason to use "remanufacture" (manus $=$ hand). All the remanufacturing processes use the synchronous interaction.

The Cordus theory provides a unified equation for nucleon remanufacture:

$$
p+2 y+i z=n+\underline{e}+v
$$

with particle identities and discrete force structures as follows:

\begin{tabular}{|c|c|c|c|}
\hline$n$ & neutron & $n\left(r_{1}^{1} \cdot a_{1}^{1} \cdot t\right)$ & $\begin{array}{l}\text { Shown for overt part. There is also } \\
\text { a large covert component }\end{array}$ \\
\hline$p$ & proton & $p\left(r_{1.1}^{1} \cdot a_{1} \cdot t_{1}\right)$ & ibid \\
\hline$e$ & electron & $e\left(r^{1} \cdot a^{1} \cdot t^{1}\right)$ & \\
\hline$\underline{e}$ & antielectron & $\underline{e}\left(r_{1} \cdot a_{1} \cdot t_{1}\right)$ & positron \\
\hline$v$ & neutrino & $v\left(r_{1}^{1} \cdot a \cdot t_{1}^{1}\right)$ & \\
\hline$\underline{v}$ & antineutrino & $\underline{v}\left(r_{\underline{1}}^{1} \cdot a \cdot t_{\underline{1}}^{\underline{1}}\right)$ & \\
\hline$y$ & photon & $y\left(r^{\uparrow} \cdot a \cdot t\right)$ & $\begin{array}{c}{ }^{\ddagger} \text { denotes oscillating discrete force, } \\
\text { extended and withdrawn }\end{array}$ \\
\hline$z$ & discrete force complex & $x_{1.1}^{1.1}$ & $\begin{array}{l}\mathrm{x} \text { is one of the emission } \\
\text { directions [r. a. } \mathrm{t}]\end{array}$ \\
\hline $2 y$ & a pair of photons & {$\left[r_{1}^{1} \cdot a_{1}^{1} \cdot t_{1}^{1}\right]$} & $\begin{array}{l}\text { With sufficient energy can also correspond } \\
\text { to an electron-antielectron pair }\end{array}$ \\
\hline$i$ & quantity, e.g. of photons & & \\
\hline
\end{tabular}

Note that antimatter is shown with underscore in this notation.

The equation works in both directions. Transfers of a particle across the equality result in inversion of the matter-antimatter species (hand). Rearrangement of the equation gives $\beta-, \beta+$, and $\mathrm{EC}$ in the conventional forward directions, and predicts induced decays too [27].

From this perspective the emission/absorption of a photon is also a remanu- 
facturing process [15], as is pair production [28], and annihilation [21]. Furthermore the asymmetrical baryogenesis and leptogensis problems have solutions with the Cordus theory [24], with the genesis remanufacturing process involving electron-antielectron pair production, with the antielectron remanufactured (with the additional of further photon discrete force structures) into the proton:

$$
\mathbf{8 y}+\boldsymbol{z} \Rightarrow e+p+2 \underline{\boldsymbol{y}}
$$

with particle identities as above. This manufacturing process was derived from consideration of the discrete force structures. It simultaneously addresses baryoand leptogenesis. Diagrammatically it is represented in Figure 9.

In summary, the weak force is reconceptualised as a remanufacturing process. The $\mathrm{W}$ and $\mathrm{Z}$ bosons are denied causal identity as vectors of change, but instead proposed to be merely transitional assembly structures. This is consistent with their short lives and ranges. Consequently we propose that the weak is not a force interaction. Nonetheless it is a powerful mechanism because it gives rise to all observed matter. Furthermore, like all the other interactions, it is based on attributes of the discrete forces, though the aggregate thereof rather than individual attributes.

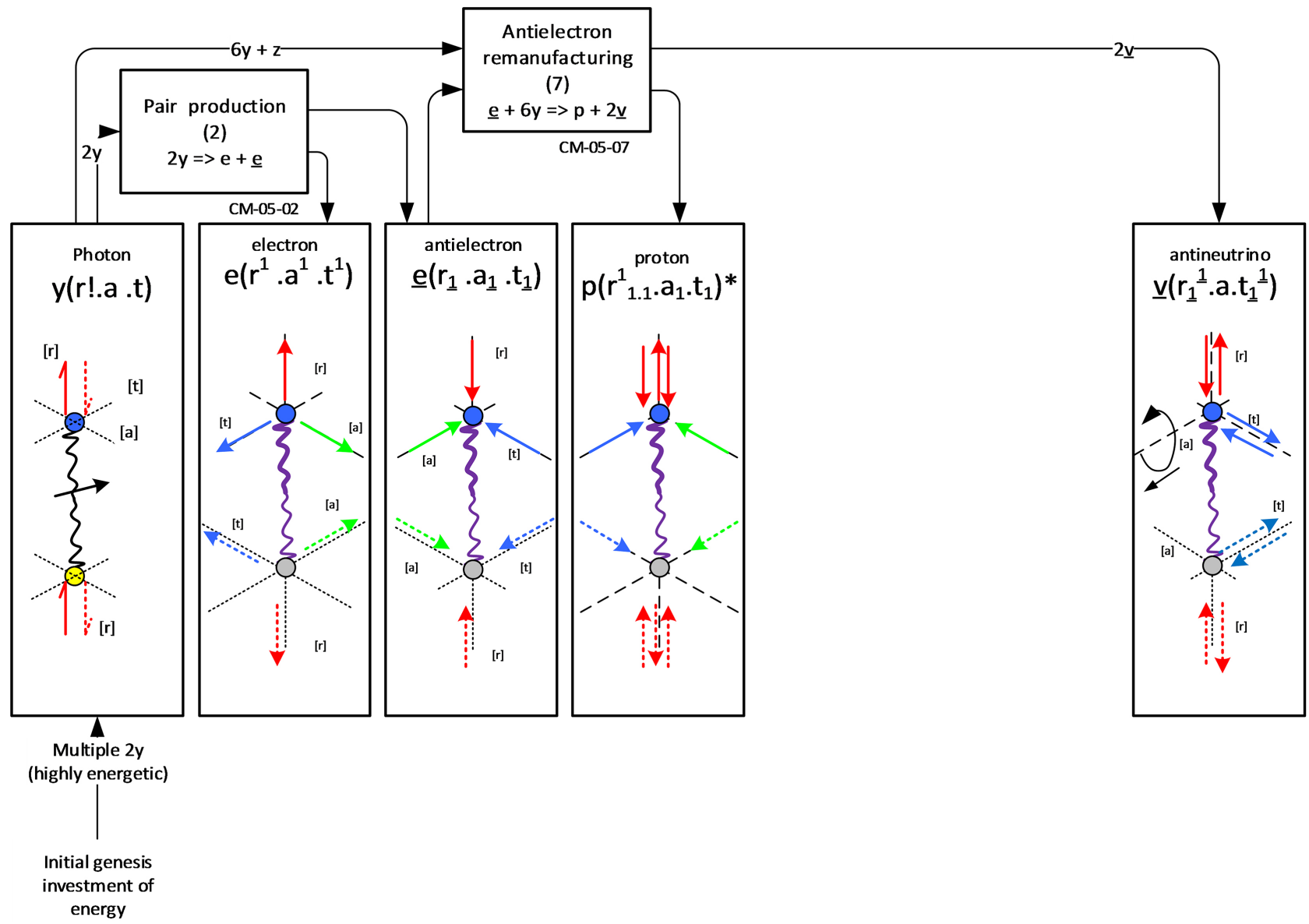

Figure 9. Asymmetrical genesis production stream. The discrete force rules predict a process whereby the antielectron from pair production is remanufactured into a proton, with two antineutrinos ejected in the waste stream. From [24]. 


\section{Discussion}

\subsection{Findings}

The results offer a new way of categorising the interactions. Thus the synchronous interaction becomes the mechanism for bonding coherent assemblies of matter, whereas the electro-magneto-gravitational interactions operate on discoherent matter. These interactions are not continuous but in discrete force increments. The remanufacturing interaction is somewhat different to the others, being less a force and more a large family of processes that change the internal assembly of multiple discrete forces and thereby change particle identity, though it too is synchronous in nature.

We find against the conventional idea that unification might be found at higher energy levels. Instead energy is only a proxy variable. The energy in an interaction between particles depends on the number of discrete force involved (hence also mass), and the type of synchronisation of emissions (discoherent or synchronous). Thus there is an approximate increase of energy involved with the progression from the electro-magneto-gravitational forces, to the synchronous, and to the remanufacturing processes with the many discrete forces involved. However we propose it is not energy per se that provides the explanation for unification.

Furthermore the theory offers an information interpretation. The emitted discrete forces communicate to other particles in the universe at large, by broadcasting the identity and attributes of the emitting particle. These attributes include position, orientation, velocity, cis/trans-phasic partnership opportunities, etc. They are a type of information broadcast that consumes no energy, yet allows other remote particles to change their behaviour.

\subsection{Contrasts}

QM attributes the electrostatic interaction to the virtual photon gauge boson. The Cordus theory instead proposes that the interaction occurs via discrete forces. In the Cordus theory there is an important difference between the single pair of discrete forces emitted by a photon, and the three orthogonal ones emitted by a massy particle [15]. The former continue to propagate outwards whereas the latter are extended and then withdrawn. Hence we disfavour identifying the interaction with a virtual photon like structure.

QM proposes that the strong force between quarks arises from the exchange of gluons, and the nuclear force arises from the residual force thereof. The QM concept of three colour charges has parallels with the three orthogonal charge emission directions of the Cordus theory, and the gluons with the discrete forces. However a key difference is that QM has different bosons for each interaction, whereas the Cordus theory attributes the interactions to different functional attributes of the same discrete forces throughout.

The Cordus perspective of gravitation emerges as being similar to but also different from General relativity (GR). In GR, gravitation arises from the curva- 
ture of spacetime, and is not so much a force as a geometric interaction of the moving body with that curvature. GR does not explain what makes up spacetime. By comparison the Cordustheory proposes the vacuum is filled with a tangle of discrete forces in their flux tube, which is called the fabric [29] [30]. Both perspectives agree that gravitation is an effect that a mass does to the whole universe. The relativistic Doppler and time dilation have also been derived from first principles using the Cordus theory [23], so there are areas of substantive alignment between the theories.

Both QM and the Cordus theory agree that gravitation is quantised. The Cordus theory offers a specific mechanism, via the discrete forces and their effect on the position of the reactive end. QM is unable to explain gravitation, and its closest approach is loop quantum gravity that proposes that the fabric consists of spin networks. A region of Cordus fabric contains multiple discrete forces in their flux tube, and conceptually these momentarily define small dynamic domains: perhaps these correspond to spin networks. However from the Cordus perspective the underlying mechanism is force lines and force pulses. Loops in the fabric are not precluded, but are interpreted as secondary phenomena rather than the mechanism itself.

\subsection{Implications}

Of the main theoretical approaches to developing a new physics to unite the interactions, quantum mechanics has been the dominant area of endeavour. While string theory is still an active area of research, it has seen less attention and been less successful in this area. The third branch, the NLHV sector, has been conceptually unproductive and become obscure. Specific solutions for the hidden structure have seemed intractable or ridiculous. Nonetheless the NLHV approach has many positive attributes, as shown here, once the question of substructure can be resolved. Demonstrating conceptually that the interactions may be unified in an NLHV theory opens up new lines of thinking.

\section{Conclusions}

The originality in this work is providing an explanation for all the interactions based on non-local hidden-variable theory. Under the assumptions of this theory, the interactions arise from different aspects of a single underlying mechanism, of the discrete force emissions.

1) The electrostatic interaction results from the direct linear operation of the discrete forces.

2) The magnetic interaction results from the bending of the flux tube containing the discrete forces.

3) Gravitation results from the handed sequence of the discrete force emission, a type of torsional effect.

4) The strong interaction (and nuclear force) arises from the synchronisation of timing of discrete force emissions between reactive ends on different particles. 
5) The weak (decay) interactions arise from the rearrangement of discrete force emissions, which results in remanufacturing of particle identity.

Apart from the concept of the discrete force, and its multiple attributes, no new particles or bosons are required.

\section{Acknowledgements}

This work builds on earlier conceptual papers [7] and [8]. The contribution made by those authors is acknowledged with gratitude.

\section{Conflict of Interest Statement}

The author declares that there are no financial conflicts of interest regarding this work.

\section{References}

[1] Pons, D.J., Pons, A.D., Pons, A.M. and Pons, A.J. (2012) Physics Essays, 25, 132-140. https://doi.org/10.4006/0836-1398-25.1.132

[2] Pons, D.J., Pons, A.D. and Pons, A.J. (2019) Journal of Modern Physics, 10, 835-860. https://doi.org/10.4236/jmp.2019.107056

[3] Smolin, L. (2015) Lessons from Einstein's 1915 Discovery of General Relativity. 1-14.

[4] Verlinde, E. (2011) Journal of High Energy Physics, 2011, 29. https://doi.org/10.1007/JHEP04(2011)029

[5] de Broglie, L. (1929) The Wave Nature of the Electron, in Nobel Lecture. Nobel Prize in Physics.

[6] Bohm, D. and Bub, J. (1966) Reviews of Modern Physics, 38, 453-469. https://doi.org/10.1103/RevModPhys.38.453

[7] Pons, D.J., Pons, A.D., Pons, A.M. and Pons, A.J. (2011) Electromagnetism. 1-17. http://vixra.org/abs/1104.0027

[8] Pons, D.J., Pons, A.D., Pons, A.M. and Pons, A.J. (2011) Gravitation, Mass and Time. 1-14. http://vixra.org/abs/1104.0029

[9] Ackoff, R.L. (1962) Scientific Method Optimising Applied Research Decisions. John Wiley \& Sons, New York.

[10] Pons, D.J., Pons, A.D. and Pons, A.J. (2013) Applied Physics Research, 5, 107-126. https://doi.org/10.5539/apr.v5n5p107

[11] Pons, D.J., Pons, A.D. and Pons, A.J. (2016) Journal of Modern Physics, 7, 1049-1067. https://doi.org/10.4236/jmp.2016.710094

[12] Pons, D.J., Pons, A.D. and Pons, A.J. (2013) Applied Physics Research, 5, 23-47. https://doi.org/10.5539/apr.v5n6p23

[13] Pons, D.J. (2020) Journal of Modern Physics, 11, 1560-1575. http://dx.doi.org/10.4236/jmp.2020.1110097

[14] Pons, D.J. (2015) Internal Structure of the Electron (Image Licence Creative Commons Attribution 4.0). Wikimedia Commons, (Creative Commons Attribution 4.0 International license). https://commons.wikimedia.org/wiki/File:Internal_structure_of_the_electron.jpg

[15] Pons, D.J. (2015) Applied Physics Research, 7, 14-26. 
https://doi.org/10.5539/apr.v7n4p24

[16] Pons, D.J., Pons, A.D. and Pons, A.J. (2014) Applied Physics Research, 6, 50-63. https://doi.org/10.5539/apr.v6n3p50

[17] Pons, D.J., Pons, A.D. and Pons, A.J. (2015) Applied Physics Research, 7, 1-11. https://doi.org/10.5539/apr.v7n1p1

[18] Sard, R.D. (1947) Electrical Engineering, 66, 61-65. https://doi.org/10.1109/EE.1947.6443336

[19] Pons, D.J., Pons, A.D. and Pons, A.J. (2014) Physics Essays, 27, 26-35. https://doi.org/10.4006/0836-1398-27.1.26

[20] Pons, D.J. (2015) Internal Structure of the Anti-Electron (Positron) (Image Licence Creative Commons Attribution 4.0). Wikimedia Commons, (Creative Commons Attribution 4.0 International License).

https://commons.wikimedia.org/wiki/File:Internal_structure_of_the_anti-electron_ (positron).jpg

[21] Pons, D.J., Pons, A.D. and Pons, A.J. (2014) Applied Physics Research, 6, 28-46. https://doi.org/10.5539/apr.v6n2p28

[22] Minkin, L. and Shapovalov, A.S. (2018) Physics Education, 53, Article ID: 035014. https://doi.org/10.1088/1361-6552/aaab88

[23] Pons, D.J., Pons, A.D. and Pons, A.J. (2018) Journal of Modern Physics, 9, 500-523. https://doi.org/10.4236/jmp.2018.93035

[24] Pons, D.J., Pons, A.D. and Pons, A.J. (2014) Journal of Modern Physics, 5, 1980-1994. https://doi.org/10.4236/jmp.2014.517193

[25] Pons, D.J., Pons, A.D. and Pons, A.J. (2015) Physics Research International, 2015, Article ID: 651361. https://doi.org/10.1155/2015/651361

[26] Pons, D.J., Pons, A.D. and Pons, A.J. (2013) Applied Physics Research, 5, 145-174. https://doi.org/10.5539/apr.v5n6p145

[27] Pons, D.J., Pons, A.D. and Pons, A.J. (2015) Applied Physics Research, 7, 1-13. http://vixra.org/abs/1412.0279 https://doi.org/10.5539/apr.v7n2p1

[28] Pons, D.J., Pons, A.D. and Pons, A.J. (2015) Journal of Nuclear and Particle Physics, 5, 58-69.

[29] Pons, D.J. and Pons, A.D. (2013) The Open Astronomy Journal, 6, 77-89. https://doi.org/10.2174/1874381101306010077

[30] Pons, D.J., Pons, A.D. and Pons, A.J. (2016) Applied Physics Research, 8, 111-121. https://doi.org/10.5539/apr.v8n3p111 